\title{
Primary malignant melanoma of the vagina in a postmenopausal woman
}

\section{Hee Young Ahn, Jin Wan Park, and Jong Soo Kim}

Department of Obstetrics and Gynecology, College of Medicine, Dankook University, Cheonan, Korea

\section{CASE STUDY}

Please cite this paper as: Ahn HY, Park JW, Kim JS. Primary malignant melanoma of the vagina in a postmenopausal woman. AMJ 2018;11(2):87-90.

https://doi.org/10.21767/AMJ.2017.3296

\section{Corresponding Author:}

Jong Soo Kim

Department of Obstetrics and Gynecology

College of Medicine, Dankook University

201 Manghyang-ro, Dongnam-gu, Cheonan 31116, Korea

Email: soo8541@hanmail.net

\section{ABSTRACT}

Primary malignant melanoma of the vagina, a very rare malignancy, is very aggressive and highly metastatic. Primary vaginal melanoma usually has a poor clinical prognosis, because it is often diagnosed at an advanced stage. We present a case of an 80-year-old postmenopausal woman with pigmented lesion of the vagina. The histopathology of the lesion revealed malignant melanoma. The patient was treated surgically, with wide local excision of the vaginal lesion. Left inguinal lymphadenectomy, based on the positron emission tomography-computed tomography (PET-CT) images, found metastatic melanoma. We present a case report of postmenopausal woman with primary vaginal melanoma.

\section{Key Words}

Malignant melanoma, postmenopause, vagina

\section{Implications for Practice:}

\section{What is known about this subject?}

Primary vaginal melanoma is very aggressive and highly metastatic. There is no consensus on what treatment modality is the best.
2. What new information is offered in this case study?

To our knowledge, this is the first case of primary vaginal melanoma in the Australasian Medical Journal.

3. What are the implications for research, policy, or practice?

This study contains general information about primary vaginal melanoma including diagnosis, treatment, and prognosis of primary vaginal melanoma.

\section{Background}

Primary malignant melanoma of the vagina is a rare and very aggressive tumour with the age-adjusted incidence of only 0.7 per million females in Asia. ${ }^{1}$ About 1.6 per cent of all melanomas arise on the female genitals. ${ }^{2}$ Primary malignant melanoma of the vagina most commonly occurs in the sixth and seventh decades of life and represents less than 3 per cent of all vaginal neoplasms. ${ }^{3,4}$ The prognosis for primary malignant melanoma of the vagina is usually very poor, because most of the cases are diagnosed at an advanced stage. For all treatment modalities, the five-year survival rate is only 8.4 per cent, according to a review of the literature. ${ }^{5}$ Currently, because primary malignant melanoma of the vagina is rare, there is no consensus on what treatment modality is the best. We report a case of vaginal primary malignant melanoma located in the upper third of the left posterolateral vaginal wall.

\section{Case details}

An 80 -year-old woman, gravida 11 , para 10 , reported that she has had vaginal bleeding for the last two weeks. She had reached menopause at the age of 50 . Her surgical history was unremarkable, and there was no history of cancer in her family. A gynaecological examination revealed a black, raised, and irregular lesion in the upper third of her left posterolateral vaginal wall (Figure 1). Bilateral parametria were not involved, and there were no palpable inguinal lymph nodes. The patient had no skin lesions that might create a suspicion of melanoma. After the preliminary biopsy was done, the histological diagnosis of malignant melanoma was confirmed by positive immunohistochemical analysis for melanoma antigen recognized by T cells (MART1) and human melanoma black-45 (HMB-45). 
Preoperative magnetic resonance imaging (MRI) of the abdomen and pelvis detected a nodular vaginal mass (Figure $2 \mathrm{~A}$ and $2 \mathrm{~B}$ ), with enhancing mass in the left superficial inguinal region (Figure $2 \mathrm{C}$ ). She had a rectal polyp diagnosed by sigmoidoscopy that proved to be a tubular adenoma. Chest radiography and cystoscopy were normal. PET-CT revealed a hypermetabolic left inguinal lymph node (Figure 2D). There was no other evidence of distant metastasis.

The patient was treated surgically with wide local excision of the vaginal lesion (Figure $3 \mathrm{~A}$ ). An enlarged left inguinal lymphadenectomy was also performed. The final diagnosis was primary malignant melanoma of the vagina with ulceration and clear resection margins of $10 \mathrm{~mm}$. The left inguinal node was revealed to be a metastatic melanoma. The tumour cell expressed HMB-45 and S100 in the immunohistochemical analysis (Figure $3 B$ and $3 C$ ). The patient refused any additional therapy. Follow-up 8 months after initial diagnosis, with CT of the abdomen and pelvis, revealed no evidence of local recurrence or distant metastasis.

\section{Discussion}

Malignant melanomas are mostly a skin disease, but may rarely occur at other sites, such as the urogenital tract, the ocular area, the nasal cavity, the oral cavity, or the perianal region. The most common site in the female genital tract, the vulvar area, accounted for 76.8 per cent, followed by the vaginal area, which accounted for 19.8 per cent; the uterine cervical area accounted for only 2.21 per cent. ${ }^{6}$ Most of cases have no early symptoms, a lack that leads to late diagnosis and then to poor prognosis. The most common symptoms include vaginal bleeding and discharge, palpable vaginal mass, and less commonly pain. Vaginal melanoma presents most commonly as a brown to black nodule, usually found in the anterior wall and lower third of the vagina. ${ }^{7}$

If a pigmented lesion is observed and suspected to be a melanoma, a biopsy should be considered. Histopathology is the gold standard for diagnosis of malignant melanoma. Immunohistochemistry is an important adjunct to routine histology and can be helpful in difficult cases. The most common and widely used markers are S100, MART-1, and HMB- 45 . $^{8}$

Vaginal melanoma is highly malignant, because it can spread hematogenously and there are abundant lymphatic plexus in the vagina. Accordingly, vaginal melanoma generally tends to recur early and locally, with metastases to the lymph nodes. In general, the upper two-thirds of the vagina drains to the obturator and iliac lymph nodes. In the lower third of the vagina, lymph drains into the inguinal and femoral lymph nodes. ${ }^{9}$ Our case suggests that she may have alterations in usual lymphatic drainage leading to atypical presentation of metastatic node.

Most vaginal cancers are staged using the International Federation of Gynaecology and Obstetrics (FIGO) system of staging combined with the American Joint Committee of Cancer (AJCC) classification. No staging system has proved to be a useful predictor of prognosis for vaginal melanoma, although tumour size has been shown to predict survival in some series. ${ }^{10,11}$

The optimal treatment of vaginal melanoma is a subject of controversy. Treatment modalities include wide local excision, radical extirpation with lymphadenectomy, and nonsurgical treatments, including radiotherapy, chemotherapy, and immunotherapy. ${ }^{3,5,10-13}$ Surgery is the mainstay of treatment for vaginal melanoma, especially for localized disease. Lymphadenectomy with or without excision of primary tumour should be considered in cases of clinically apparent nodal disease. Postoperative immunotherapy can be a treatment modality for patients with vaginal melanoma. ${ }^{14}$ There is no effective systemic therapy for vaginal melanoma. Intravaginal brachytherapy should be combined for vaginal melanoma. ${ }^{15}$ For the patients with an advanced stages, palliative chemotherapy is preferred. ${ }^{16}$

The prognosis for vaginal melanoma is usually very poor. The size of the tumour significantly affects median survival. Patients with a tumour of less than $3 \mathrm{~cm}$ had a median survival of 41 months, whereas survival was only 12 months for those with a tumour of $3 \mathrm{~cm}$ or more. ${ }^{10}$ Patients with lymph node involvement had a worse overall survival rate than those with disease localized in the vagina. ${ }^{17}$ The local recurrence following surgery was reported to be up to 40 per cent, with high risk for distant metastases. ${ }^{7}$

According to the staging system, our patient was diagnosed with stage III melanoma because of the presence of lymph node metastasis. She underwent wide local excision with left inguinal lymphadenectomy. Postoperative radiotherapy or immunotherapy was recommended to her, but she refused any additional therapy.

Because of the rarity of vaginal melanoma, and because relatively little is known about its pathogenesis and risk factors, there are no well-established protocols for staging and treatment of the disease. Further studies are needed to 
make the guidelines and protocols specific to vaginal melanoma.

\section{Conclusion}

Primary malignant melanoma of the vagina, a very rare malignancy, is very aggressive and highly metastatic. Primary vaginal melanoma usually has a poor prognosis, because it is often diagnosed at an advanced stage. If a lesion is suspected to be a melanoma, we have to take a biopsy. Unfortunately, because primary vaginal melanoma is rare, there is no consensus on what treatment modality is the best. We described a very rare case of a postmenopausal woman with primary vaginal melanoma. This study contains general information about the disease including diagnosis, treatment, and prognosis of primary vaginal melanoma.

\section{References}

1. Hu DN, Yu GP, McCormick SA. Population-based incidence of vulvar and vaginal melanoma in various races and ethnic groups with comparisons to other sitespecific melanomas. Melanoma Res. 2010;20:153-158. doi: 10.1097/CMR.0b013e32833684e8.

2. Miner TJ, Delgado R, Zeisler J, et al. Primary vaginal melanoma: a critical analysis of therapy. Ann Surg Oncol. 2004;11:34-39.

3. Baloglu A, Bezircioglu I, Cetinkaya B, et al. Primary malignant melanoma of the vagina. Arch Gynecol Obstet. 2009;280:819-822. doi: 10.1007/s00404-009-1009-0.

4. Piura B. Management of primary melanoma of the female urogenital tract. Lancet Oncol. 2008;9:973-981. doi: 10.1016/S1470-2045(08)70254-7.

5. Irvin WP, Bliss SA, Rice LW, et al. Malignant melanoma of the vagina and locoregional control: radical surgery revisited. Gynecol Oncol. 1998;71:476-480. doi: 10.1006/gyno.1998.5188.

6. McLaughlin $\mathrm{CC}, \mathrm{Wu} \mathrm{XC}$, Jemal $\mathrm{A}$, et al. Incidence of noncutaneous melanomas in the U.S. Cancer. 2005;103:1000-1007. doi: 10.1002/cncr.20866.

7. Reid GC, Schmidt RW, Roberts JA, et al. Primary melanoma of the vagina: a clinicopathologic analysis. Obstet Gynecol. 1989;74:190-199.

8. Ohsie SJ, Sarantopoulos GP, Cochran AJ, et al. Immunohistochemical characteristics of melanoma. J Cutan Pathol. 2008;35:433-444. doi: 10.1111/j.16000560.2007.00891.x.

9. Hacker NF, Eifel PJ, van der Velden J. Cancer of the vagina. Int J Gynaecol Obstet. 2015;131:S84-87. doi: 10.1016/j.ijgo.2015.06.003.

10. Buchanan DJ, Schlaerth J, Kurosaki T. Primary vaginal melanoma: thirteen-year disease-free survival after wide local excision and review of recent literature. Am J Obstet Gynecol. 1998;178:1177.

11. Petru E, Nagele F, Czerwenka K, et al. Primary malignant melanoma of the vagina: long-term remission following radiation therapy. Gynecol Oncol. 1998;70:23-26. doi: 10.1006/gyno.1998.4982

12. Moodley M, Daya M, Moodley J. Vaginal malignant melanoma: a case report and literature review. Int J Gynecol Cancer. 2004;14:687-689. doi: 10.1111/j.1048891X.2004.14430.x.

13. Gökaslan H, Şişmanoğlu A, Pekin $T$, et al. Primary malignant melanoma of the vagina: a case report and review of the current treatment options. Eur J Obstet Gynecol Reprod Biol. 2005;121:243-248. doi: 10.1016/j.ejogrb.2004.11.048.

14. Huang $Q$, Huang $H$, Wan $T$, et al. Clinical outcome of 31 patients with primary malignant melanoma of the vagina. J Gynecol Oncol. 2013;24:330-335. doi: 10.3802/jgo.2013.24.4.330.

15. Leitao MM, Cheng $X$, Hamilton AL, et al. Gynecologic cancer intergroup (GCIG) consensus review for vulvovaginal melanomas. Int J Gynecol Cancer. 2014;24:S117-122. doi: 10.1097/IGC.0000000000000198.

16. Kalampokas E, Kalampokas T, Damaskos C. Primary vaginal melanoma, a rare and aggressive entity. A case report and review of the literature. In Vivo. 2017;31:133-139. doi: 10.21873/invivo.11036.

17. Kirschner AN, Kidd EA, Dewees $T$, et al. Treatment approach and outcomes of vaginal melanoma. Int J Gynecol Cancer. 2013;23:1484-1489. doi: 10.1097/IGC.0b013e3182a1ced8.

\section{PEER REVIEW}

Not commissioned. Externally peer reviewed.

\section{CONFLICTS OF INTEREST}

The authors declare that they have no competing interests.

\section{FUNDING}

None

\section{PATIENT CONSENT}

The authors, Ahn HY, Park JW, Kim JS, declare that:

1. They have obtained written, informed consent for the publication of the details relating to the patient in this report.

2. All possible steps have been taken to safeguard the identity of the patient.

3. This submission is compliant with the requirements of local research ethics committees. 
Figure 1: Preoperative view of vaginal mass, with a black and raised lesion in the upper third of the vagina

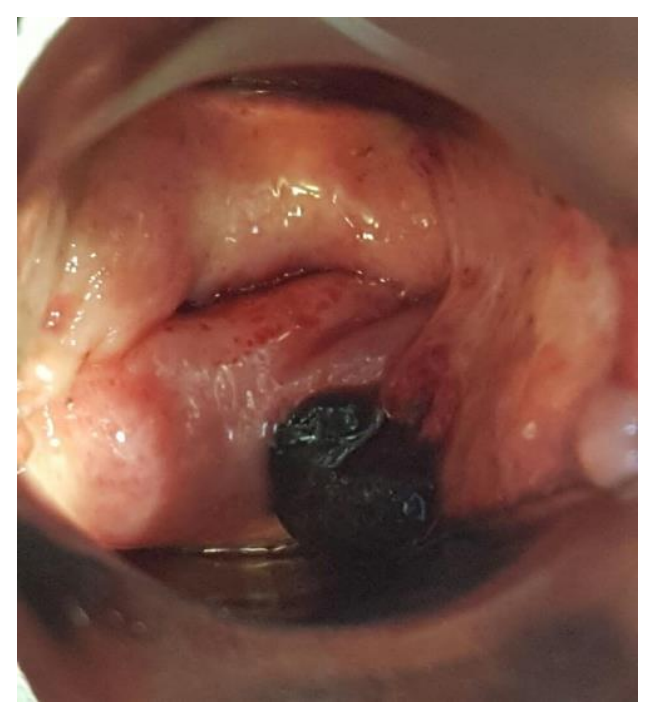

Figure 2: Magnetic resonance imaging findings. (A) MRI shows a nodular high signal intensity lesion (arrow) in the left aspect of the upper third of the vagina measuring approximately $1.0 \times 0.3 \mathrm{~cm}$ on T1WI. (B) On T2WI, a vertically elongated lesion with intermediate to low signal intensity measuring $1.0 \times 0.3 \mathrm{~cm}$ (arrow) is seen in the upper third of the vagina. (C) There is a $2.3 \times 2.1 \mathrm{~cm}$ enlarged lymph node in the left inguinal region on T2WI. (D) PET-CT shows a $2.3 \times 2.1 \mathrm{~cm}$ lymph node with intense FDG uptake in the left inguinal region
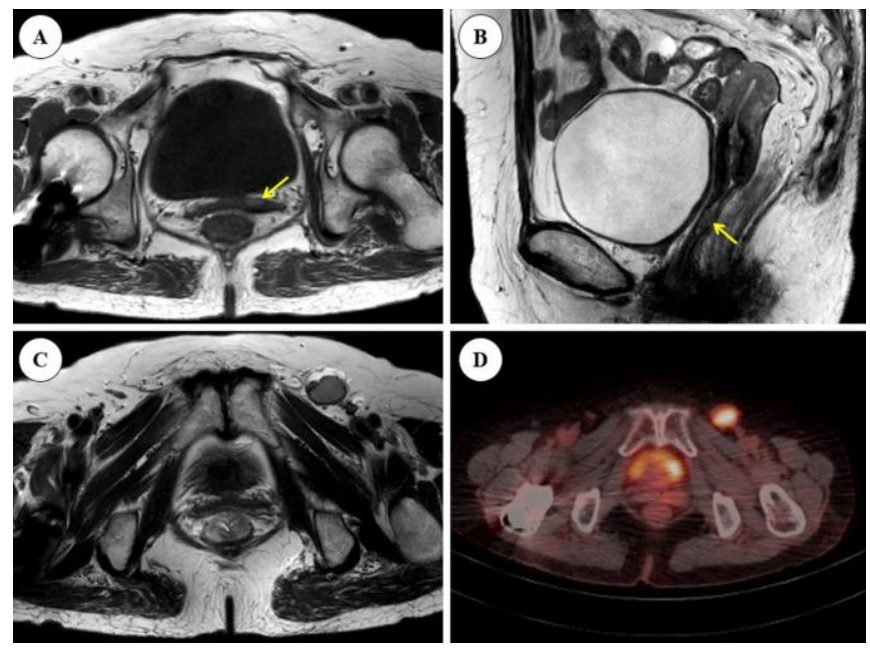

Figure 3: (A) Gross appearance of the vaginal melanoma, measuring $2.3 \times 1.5 \times 0.5 \mathrm{~cm}$ with clear resection margins. (B) The immunohistochemical staining of the malignant melanoma for presence of HMB-45 $(\times 200)$. (C) The malignant cells were included brown granules of melanin pigments (Hematoxylin and eosin stain, $\times 400$ )
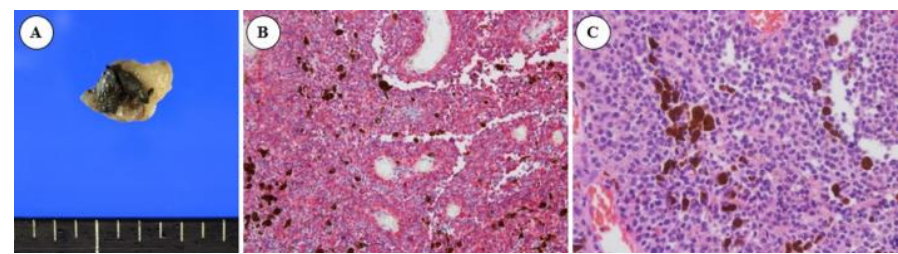